\title{
Avaliação das Propriedades Energéticas de Resíduos de Madeiras Tropicais com Uso da Espectroscopia NIR
}

\author{
Dimas Agostinho da Silva ${ }^{1}$, Vanessa Coelho Almeida ${ }^{1}$, \\ Lívia Cássia Viana ${ }^{1}$, Umberto Klock ${ }^{1}$, Graciela Inez Bonzon de Muñiz ${ }^{1}$ \\ ${ }^{1}$ Departamento de Engenharia e Tecnologia Florestal, Universidade Federal do Paraná - UFPR, Curitiba/PR, Brasil
}

\begin{abstract}
RESUMO
O objetivo deste trabalho foi avaliar o potencial de resíduos de madeira de quatro espécies amazônicas para fins energéticos, além de calibrar propriedades da madeira e do carvão vegetal utilizando a técnica NIRS. Foram utilizadas costaneiras das espécies cardeiro (Scleronema micranthum Ducke), cedrinho (Scleronema sp.), louro (Ocotea sp.) e piquiarana (Caryocar villosum Pers.), tendo sido determinadas as seguintes propriedades: densidade básica, composição química, análise química imediata e poder calorífico superior da madeira; rendimentos em carvão vegetal; análise química imediata, poder calorífico e friabilidade do carvão vegetal. Foram adquiridos espectros NIR e gerados modelos para calibração de cada propriedade energética. Os resultados indicam que todas as espécies possuem potencial como fonte bioenergética. Entretanto, as melhores espécies foram o cardeiro e o piquiarana, devido à maior densidade básica e energética, além do louro, com maior teor de lignina e carbono fixo, que apresentou, consequentemente, maior poder calorífico. A espectroscopia NIR mostrou-se como uma ferramenta possível para calibração de propriedades energéticas da biomassa.
\end{abstract}

Palavras-chave: espécies nativas, resíduos de florestais, pirólise, infravermelho próximo.

\section{Evaluation of the Energy-related Properties of Tropical Wood Waste Using NIR Spectroscopy}

\begin{abstract}
The aim of this study was to evaluate the energy-related potential of wood residues of four Amazonian species and calibrate the properties of wood and charcoal using the Near Infrared Spectrometry (NIRS) technique. Residue slabs of cardeiro (Scleronema micranthum Ducke), cedrinho (Scleronema sp.), louro (Ocotea sp.), and piquiarana (Caryocar villosum Pers.) wood were used. The following properties were determined: density, chemical composition, immediate chemical analysis, and superior calorific value of wood; and yield, immediate chemical analysis, calorific value, and friability of charcoal. NIR spectra were obtained and calibration models were generated for each energy property. The results indicate that residues of these forest species can be used as a renewable energy source. NIR spectroscopy has proved to be a possible tool for calibrating energy properties of wood.
\end{abstract}

Keywords: native species, forestry residues, pyrolysis, near infrared. 


\section{INTRODUÇÃO E OBJETIVOS}

Embora os estudos sobre as espécies florestais nativas sejam de interesse há muitos anos, pouco se sabe sobre as propriedades físicas, químicas e mecânicas da maioria das espécies nativas da Amazônia.

Dentre as propriedades físico-químicas a serem avaliadas, destacam-se as energéticas. O bioma Amazônico apresenta espécies florestais ainda pouco estudadas para fins energéticos, embora a atividade de produção de energia a partir da madeira seja amplamente difundida, principalmente para uso doméstico e para produção de carvão para indústrias siderúrgicas, como acontece, por exemplo, no estado do Pará.

Sob regime de Plano de Manejo Florestal Sustentável, é possível viabilizar a exploração florestal otimizando o aproveitamento da madeira, inclusive com a utilização dos resíduos, contemplando a sustentabilidade dos recursos naturais.

O melhor rendimento energético da biomassa madeira depende de sua constituição química, entre outras propriedades. Os teores de celulose, hemiceluloses, lignina, extrativos e substâncias minerais variam com a espécie, e são de grande importância para a escolha adequada da madeira a ser utilizada.

Com relação à composição química da madeira, de acordo com Trugilho \& Silva (2001) e Vindaurre et al. (2012), a presença de alto teor de substâncias de natureza aromática, tais como alguns extrativos e lignina, gera, como consequência, um carvão com maior densidade e mais resistente, em termos de propriedades físico-mecânicas.

A lignina é um componente estrutural que confere propriedades de rigidez e resistência à madeira. O conhecimento do teor deste componente é fundamental para produção de energia e carvão vegetal, pois a lignina apresenta alto poder calorífico $(6.100 \mathrm{kcal} / \mathrm{kg})$ e há fortes evidências de que contribui significativamente para a formação do carbono residual, devido à sua estrutura complexa. Este fato está relacionado à sua alta resistência à degradação térmica, quando comparada à holocelulose, principalmente devido ao maior número de ligações $\mathrm{C}-\mathrm{C}$ e $\mathrm{C}=\mathrm{C}$ presentes em sua estrutura, como também à maior presença de carbono elementar e ao baixo teor de oxigênio, em comparação com as outras substâncias químicas componentes da madeira, (Petroff \& Doat, 1978; Pereira et al., 2012).

Pastore et al. (1989) encontraram correlação positiva entre os teores de lignina e extrativos, e o rendimento gravimétrico em carvão de 20 espécies tropicais da Região Amazônica. O rendimento gravimétrico em carvão vegetal está correlacionado positivamente com o teor de materiais voláteis e negativamente com o teor de carbono fixo, conforme encontrado em estudos de espécies de cerrado e de eucalipto (Protásio et al., 2011, 2012; Vale et al., 2002).

O conhecimento das propriedades da madeira é de fundamental importância para a indicação dos potenciais de utilização final deste material. Conforme já ressaltado anteriormente, a qualidade do carvão vegetal é influenciada principalmente pela composição química da madeira que o originou, além do sistema de produção utilizado. Dessa forma, torna-se fundamental a avaliação dessas propriedades por meio de ensaios laboratoriais, destrutivos e não destrutivos.

No campo de ensaios não destrutivos, a técnica da espectroscopia na faixa do infravermelho próximo (NIRS) mostra-se como uma ferramenta rápida, que permite análises em tempo real e resultados confiáveis. O método consiste na aquisição de espectros e na consequente correlação dos mesmos com resultados de análises destrutivas, para a geração de um modelo estatístico que explique e correlacione a maioria das informações contidas nos espectros (Williams \& Norris, 2001).

A técnica da espectroscopia no infravermelho próximo (NIRS) já é conhecida e aplicada na produção e no controle de qualidade de produtos no setor agrícola, na indústria de alimentos, na indústria de polímeros e têxtil, e na produção de combustível fóssil, biocombustível, cosméticos e tintas, além da indústria farmacêutica. No setor florestal, a técnica é utilizada na estimativa de um grande número de características da madeira, referentes às propriedades físicas (Schimleck et al., 1999), químicas (Meder et al., 1999), mecânicas (Kelley et al., 2005) e anatômicas (Schimleck \& Evans, 2004).

O objetivo desta pesquisa foi caracterizar os resíduos da madeira de quatro espécies do bioma 
amazônico para fins energéticos, além de utilizar a técnica NIR para calibrações das propriedades da madeira e do carvão vegetal.

\section{MATERIAL E MÉTODOS}

\subsection{Material}

Para o presente estudo, utilizaram-se resíduos de madeira das seguintes espécies: cardeiro (Scleronema micranthum Ducke, Bombacaceae), cedrinho (Scleronema sp., Bombacaceae), louro (Ocotea sp, Lauraceae) e piquiarana (Caryocar villosum Pers., Caryocaraceae), provenientes do município de Itacoatiara-Amazonas. Os resíduos foram coletados e identificados por pesquisadores do Instituto Nacional de Pesquisas da Amazônia (INPA), Manaus-AM, para estudos tecnológicos.

O preparo das amostras, no Laboratório de Energia de Biomassa Florestal, foi realizado mediante confecção de corpos de prova nas dimensões $1,5 \times$ $1,5 \times 3,0 \mathrm{~cm}$, para o procedimento de pirólise. Para as análises da madeira, os resíduos tiveram suas dimensões reduzidas em um picador de discos e em um moinho tipo Wiley. Posteriormente, as partículas foram classificadas em peneiras de 40/60 mesh.

\subsection{Caracterização dos resíduos de madeira}

Densidade básica: As amostras das diferentes espécies foram colocadas em dessecador com água, sendo realizada a aplicação de vácuo, a fim de proceder à saturação das fibras para, em seguida, calcular o volume imerso em água dos corpos de prova. Posteriormente, foram levados para uma estufa de circulação forçada de ar na temperatura de $105 \pm 2^{\circ} \mathrm{C}$, até a massa constante de madeira, obtendose, assima a massa seca. A densidade básica das madeiras foi determinada de acordo com a norma NBR 11941-02 (ABNT, 2003).

Componentes químicos: As amostras foram preparadas de acordo com a norma NBR 14660 (ABNT, 2004). O teor de lignina insolúvel em ácido foi determinado conforme a norma NBR 7989 (ABNT, 2010a). O teor de extrativos foi determinado através da solubilidade em etanol/tolueno, de acordo com a norma NBR 14853 (ABNT, 2010b). O percentual de holocelulose foi obtido somando-se os percentuais de lignina e extrativos, e subtraindo-se este valor de $100 \%$.

Análise química imediata: A composição química imediata foi determinada de acordo com a norma NBR 8112 (ABNT, 1983), com determinação dos teores de umidade (na base seca), de materiais voláteis, do teor de cinzas e do teor de carbono fixo, em base seca.

Poder calorífico superior: foi determinado por meio de um calorímetro adiabático, de acordo com a norma NBR 8633 (ABNT, 1983).

Densidade energética: foi estimada com base na Equação 1.

$\mathrm{DE}=\mathrm{DB} \times \mathrm{PC} / 1000$

Em que:

$\mathrm{DE}=$ densidade energética expressa em $\mathrm{Mcal} / \mathrm{m}^{3}$;

$\mathrm{DB}=$ densidade básica expressa $\mathrm{em} \mathrm{Kg} / \mathrm{m}^{3}$;

$\mathrm{PC}=$ poder calorífico superior expresso em $\mathrm{Kcal} / \mathrm{Kg}$.

\subsection{Pirólise dos resíduos de madeira e caracterização do carvão vegetal}

As pirólises foram realizadas em uma mufla adaptada com reator e sistema coletor de bio-óleos, e queima dos gases não condensáveis. A temperatura final foi de $450^{\circ} \mathrm{C}$ por sete horas, com taxa de aquecimento de $1^{\circ} \mathrm{C} / \mathrm{min}$, em quatro repetições. Em cada repetição, foram utilizados 15 corpos de prova de cada espécie, totalizando 60 corpos de prova por pirólise.

O rendimento em carvão vegetal foi determinado em relação à madeira seca e o rendimento em carbono fixo foi obtido conforme a Equação 2.

$\mathrm{RCF}=(\mathrm{RGC} \times \mathrm{TCF}) / 100$

Em que:

$\mathrm{RCF}=$ rendimento em carbono fixo (\%);

$\mathrm{RGC}=$ rendimento gravimétrico em carvão (\%);

$\mathrm{TCF}=$ teor de carbono fixo (\%).

A caracterização do carvão vegetal produzido foi realizada em triplicata e compreendeu as seguintes análises: análise química imediata e o poder calorífico superior, conforme as mesmas normas utilizadas para madeira. 
Também foi determinada a friabilidade do carvão, conforme o teste de tamboramento das normas MB 1375/80 (adaptada) e NBR 8740/85. Os corpos de prova de carvão, separados por espécies, foram depositados em um tambor rotativo de 30 $\mathrm{cm}$ diâmetro e $25 \mathrm{~cm}$ de comprimento, com rotação de $30 \mathrm{rpm}$, durante 16,5 minutos. Após o ensaio, a porcentagem de finos com dimensões inferiores a 13 $\mathrm{mm}$ foi quantificada.

\subsection{Espectroscopia no infravermelho próximo}

\subsubsection{Aquisição dos espectros NIR}

Para a aquisição dos espectros, foram usados três corpos de prova por espécie, totalizando 12 amostras, com as dimensões de $1,5 \times 1,5 \times 3,0 \mathrm{~cm}$. As amostras foram previamente acondicionadas em câmara de climatização à temperatura de $20 \pm 1^{\circ} \mathrm{C}$ e umidade relativa de $65 \pm 5 \%$. Para cada amostra, foram realizadas duas leituras, sendo uma na face transversal e outra na face longitudinal tangencial.

Os espectros no infravermelho próximo foram adquiridos no espectrômetro Bruker-Tensor 37, em conjunto com o programa OPUS versão 4.2, no Laboratório de Anatomia e Qualidade da Madeira da UFPR. A aquisição espectral foi realizada na faixa de radiação de 9.000 a $4.000 \mathrm{~cm}^{-1}$, com resolução espectral de $8 \mathrm{~cm}^{-1}$. O espectro de cada amostra foi obtido por meio da média de 64 varreduras. Os espectros de absorbância adquiridos de cada amostra de madeira foram posteriormente correlacionados com os valores encontrados nos ensaios pelos métodos tradicionais para as propriedades da madeira e do carvão vegetal. Para cada propriedade, foi gerado um modelo de calibração a partir dos 12 espectros coletados das amostras das quatro diferentes espécies.

\subsection{Análise estatística}

\subsubsection{Delineamento e análise estatística}

Para a caracterização energética das quatro espécies amazônicas, foi adotado o delineamento inteiramente casualizado com quatro tratamentos (espécies) e três repetições, exceto para o poder calorífico, para o qual foram necessárias apenas duas repetições. Atendidos os pressupostos da ANOVA, os tratamentos foram comparados entre si por meio do teste de Tukey no nível de significância de 5\%.

\subsubsection{Calibração, validação e seleção dos modelos no NIR}

A análise estatística para calibração, validação e seleção dos modelos no NIR foi realizada usando-se o programa The Unscrambler Versão 9.2. A regressão dos mínimos quadrados parciais (PLS-1) e o método de validação cruzada completa foram utilizados para desenvolvimento dos modelos (Martens \& Naes, 1996; Brereton, 2000).

Outliers foram detectados utilizando-se a variância residual das amostras individuais. Os dados espectrais originais obtidos das amostras de madeira também foram transformados matematicamente pelo tratamento da primeira derivada de "SavitzkyGolay" (Savitzky \& Golay, 1964).

Os melhores modelos de predição foram então selecionados, levando-se em consideração os seguintes critérios: coeficiente de correlação do modelo na validação cruzada (Rcv); erro padrão da validação cruzada (SECV); número de fatores PLS utilizados na calibração, e a relação de desempenho do desvio (RPD) e o número de fatores PLS. A decisão do número de fatores PLS baseou-se na escolha de modelos com menor número de fatores e menores valores de erros na calibração e da validação cruzada (full cross-validation).

\section{RESULTADOS E DISCUSSÃO}

\subsection{Caracterização dos resíduos de madeira}

Na Tabela 1, estão apresentados os valores médios de densidade aparente, poder calorífico, carbono fixo, materiais voláteis, cinzas, lignina, extrativos e holocelulose da madeira das quatro espécies, além do teste de comparação múltipla realizado.

Observa-se que houve diferença significativa entre os valores médios de densidade básica da madeira. A densidade básica não apresentou relação direta com a quantidade de energia na madeira, pois madeira mais densa, como cardeiro e piquiarana, não tiveram poder calorífico maior, uma vez que o poder calorífico foi igual entre as espécies. Entretanto, ao se comparar a densidade energética, observa-se que 
há efeito positivo direto da densidade básica, pois as espécies mais densas corroboram para maior densidade energética, que é a expressão de energia por unidade de volume. Resultados semelhantes foram encontrados por Vindaurre et al. (2012) e Vale et al.(2010). O louro, com maior teor de lignina e de carbono fixo, possui maior poder calorífico. Este comportamento foi observado por Protásio et al. (2011, 2012) e Vale et al. (2002). Contudo, o louro apresenta-se com menor densidade energética devido à sua menor densidade básica, o que está de acordo com Vale et al. (2010). Os valores médios observados nas espécies estão de acordo com as pesquisas de Foelkel (2012), Trugilho \& Silva (2001) e Trugilho et al. (1997). O maior teor de lignina permite esperar melhor expressão energética das espécies, devido ao seu elevado teor de carbono (Petroff \& Doat, 1978; Pereira et al., 2012).

\subsection{Pirólise dos resíduos de madeira e caracterização do carvão vegetal}

Na Tabela 2, estão apresentados os valores médios do rendimento e das propriedades do carvão produzido com os resíduos das quatro espécies, além do teste de comparação múltipla realizado.

Observa-se, na Tabela 2, que, com exceção do poder calorífico e do teor de cinzas do carvão vegetal, não houve efeito significativo de espécie para as demais propriedades estudadas. O rendimento gravimétrico em carvão vegetal e em carbono fixo, teor de carbono fixo e material volátil, e a friabilidade foram estatisticamente iguais entre as espécies. Estes valores estão de acordo com os encontrados em literatura para diversas espécies nativas ou exóticas, conforme mencionados por Oliveira et al. (2010) e Silva (2007).

Tabela 1. Valores médios de densidade básica, umidade base seca, teor de lignina, extrativos e holocelulose das espécies e dos parâmetros energéticos da madeira.

Table 1. Mean values of density, dry basis moisture, lignin content, extractives, holocellulose and the energy parameters of the wood.

\begin{tabular}{lcccc}
\multicolumn{1}{c}{ Espécie } & Cardeiro & Cedrinho & Louro & Piquiarana \\
\hline Densidade básica $\left(\mathrm{g} / \mathrm{cm}^{3}\right)$ & $0,614 \mathrm{a}$ & $0,559 \mathrm{~b}$ & $0,571 \mathrm{~b}$ & $0,645 \mathrm{a}$ \\
\hline Poder calorífico $(\mathrm{Kcal} / \mathrm{Kg})$ & $4608 \mathrm{a}$ & $4611 \mathrm{a}$ & $4928 \mathrm{a}$ & $4785 \mathrm{a}$ \\
\hline Densidade energética $\left(\mathrm{Mcal} / \mathrm{cm}^{3}\right)$ & $2829 \mathrm{a}$ & $2578 \mathrm{~b}$ & $2814 \mathrm{a}$ & $3086 \mathrm{a}$ \\
Umidade base seca (\%) & $14,22 \mathrm{a}$ & $12,34 \mathrm{a}$ & $13,27 \mathrm{a}$ & $12,16 \mathrm{a}$ \\
\hline Carbono Fixo (\%) & $16,99 \mathrm{~b}$ & $17,73 \mathrm{~b}$ & $18,94 \mathrm{a}$ & $17,14 \mathrm{~b}$ \\
\hline Materiais Voláteis (\%) & $82,76 \mathrm{a}$ & $81,71 \mathrm{bc}$ & $80,94 \mathrm{c}$ & $82,40 \mathrm{ab}$ \\
Teor de cinzas (\%) & $0,248 \mathrm{~b}$ & $0,562 \mathrm{a}$ & $0,119 \mathrm{c}$ & $0,461 \mathrm{a}$ \\
Lignina (\%) & $27,03 \mathrm{ab}$ & $25,80 \mathrm{~b}$ & $33,64 \mathrm{a}$ & $25,13 \mathrm{~b}$ \\
Extrativos totais (\%) & $3,55 \mathrm{c}$ & $3,65 \mathrm{c}$ & $7,68 \mathrm{a}$ & $6,27 \mathrm{~b}$ \\
\hline Holocelulose $\%)$ & $69,42 \mathrm{a}$ & $70,55 \mathrm{a}$ & $58,68 \mathrm{~b}$ & $68,60 \mathrm{a}$ \\
\hline
\end{tabular}

Médias seguidas de mesma letra minúscula ao longo da mesma linha não diferem entre si pelo teste $\mathrm{F}$ com um nível nominal de significância de $5 \%$.

Tabela 2. Valores médios das propriedades energéticas do carvão.

Table 2. Mean values of the energetic properties of the charcoal.

\begin{tabular}{lcccc}
\multicolumn{1}{c}{ Espécie } & Cardeiro & Cedrinho & Louro & Piquiarana \\
Poder Calorífico (Kcal/Kg) & $7295 \mathrm{~b}$ & $6554 \mathrm{~b}$ & $8719 \mathrm{a}$ & $7394 \mathrm{~b}$ \\
Carbono Fixo (\%) & $71,47 \mathrm{a}$ & $69,54 \mathrm{a}$ & $71,13 \mathrm{a}$ & $67,72 \mathrm{a}$ \\
Materiais Voláteis (\%) & $27,0 \mathrm{a}$ & $28,8 \mathrm{a}$ & $28,7 \mathrm{a}$ & $30,8 \mathrm{a}$ \\
Teor de Cinzas (\%) & $1,550 \mathrm{a}$ & $1,673 \mathrm{a}$ & $0,223 \mathrm{~b}$ & $1,497 \mathrm{a}$ \\
Rendimento Carbono Fixo (\%) & $21,62 \mathrm{a}$ & $23,19 \mathrm{a}$ & $23,07 \mathrm{a}$ & $21,72 \mathrm{a}$ \\
Rendimento Carvão Vegetal (\%) & $29,61 \mathrm{a}$ & $32,56 \mathrm{a}$ & $32,33 \mathrm{a}$ & $31,38 \mathrm{a}$ \\
\hline Friabilidade menor 10 mm (\%) & $3,80 \mathrm{a}$ & $5,81 \mathrm{a}$ & $2,70 \mathrm{a}$ & $5,28 \mathrm{a}$ \\
\hline
\end{tabular}

Médias seguidas de mesma letra minúscula ao longo da mesma linha não diferem entre si pelo teste $\mathrm{F}$ com um nível nominal de significância de 5\%. 
O carvão do louro (Ocotea sp.) se comportou de modo semelhante à madeira que lhe deu origem, apresentando o menor teor de cinzas e o maior poder calorífico, quando comparado às outras espécies estudadas. Oliveira et al. (2010) e Vale et al. (2002) encontraram resultados semelhantes após a pirólise de madeira E. pellita e E. grandis, respectivamente.

De modo geral, os carvões produzidos apresentaram baixa porcentagem de finos, ou seja, baixa friabilidade. Tal propriedade indica que, independentemente da espécie florestal estudada, os carvões podem ser manuseados sem grandes perdas na forma de partículas ou pó. O grau de friabilidade não ultrapassou o valor numérico de 5,81\%, valor este obtido para a espécie Scleronema sp (cedrinho). De acordo com Oliveira et al. (1982), o carvão vegetal obtido é classificado como muito pouco friável, ou seja, com a quantidade de finos gerados inferior a $10 \%$.

\subsection{Espectroscopia no infravermelho próximo}

Para a predição das características da madeira e do carvão, foram realizadas calibrações com espectros NIR das faces transversal e tangencial das amostras de madeira. Contudo, nas tabelas que se seguem, serão apresentados somente os melhores ajustes, que foram conseguidos utilizando-se a face tangencial.
As calibrações NIRS para a estimativa das propriedades da madeira estão apresentadas na Tabela 3 .

Os melhores resultados encontrados para estimar as propriedades da madeira foram o teor de lignina e o poder calorífico, com valores de coeficiente de correlação na validação iguais a 0,93 e 0,92 . Estes valores, semelhantes para as características em questão, confirmam a alta correlação existente entre teor de lignina e o poder calorífico na madeira.

Para obter tais valores, foram utilizados modelos com fatores PLS variando de quatro a oito. Uma amostra outlier foi excluída para a calibração dos teores de materiais voláteis e carbono fixo. De acordo com Schimleck et al. (2003), calibrações com a relação de desempenho do desvio (RPD) superior a 2,5 são consideradas satisfatórias para a seleção de árvores em programas de melhoramento, embora valores de RPD iguais ou superiores a 1,5 sejam suficientes para leituras iniciais. Os valores de RPD obtidos variaram de 1,4 para o teor de materiais voláteis a 2,6 para o teor de lignina.

$\mathrm{Na}$ Tabela 4, são apresentados os dados referentes às calibrações realizadas para prever as características energéticas do carvão.

As calibrações para as características do carvão, utilizando-se até sete fatores PLS, geraram modelos com coeficientes de correlação na validação cruzada variando de 0,84 para teor de materiais voláteis a 0,92 para poder calorífico. Os valores do RPD foram de 1,7 a 2,3 .

Tabela 3. Calibrações NIRS para propriedades da madeira.

Table 3. NIRS calibration for wood properties.

\begin{tabular}{lccccccc}
\multicolumn{1}{c}{ Propriedade } & Rc & Rcv & SEC & SECV & Fatores & Outlier & RPD \\
\hline Lignina (\%) & 0,97 & 0,93 & 0,8436 & 1,3649 & 5 & 0 & 2,6 \\
Poder Calorífico (Kcal/kg) & 0,99 & 0,92 & 16,6998 & 56,8547 & 8 & 0 & 2,4 \\
Materiais voláteis (\%) & 0,92 & 0,74 & 0,3278 & 0,5837 & 5 & 1 & 1,4 \\
Carbono Fixo (\%) & 0,93 & 0,85 & 0,3118 & 0,4752 & 4 & 1 & 1,8 \\
\hline
\end{tabular}

$\mathrm{Rc}=$ coeficiente de correlação da calibração; $\mathrm{Rcv}=$ coeficiente de correlação da validação cruzada; $\mathrm{SEC}$ = erro padrão da calibração; SECV = erro padrão da validação cruzada; $\mathrm{RPD}=$ relação de desempenho do desvio.

Tabela 4. Calibrações NIRS para características do carvão.

Table 4. NIRS calibration for charcoal characteristics.

\begin{tabular}{lccrrrrcc}
\multicolumn{1}{c}{ Propriedade } & Rc & Rcv & \multicolumn{1}{c}{ SEC } & \multicolumn{1}{c}{ SECV } & Fatores & Outlier & RPD \\
\hline Poder Calorífico (kcal/kg) & 0,97 & 0,92 & 191,078 & 358,930 & 6 & 0 & 2,3 & 1,7 \\
Materiais Voláteis (\%) & 0,99 & 0,84 & 0,299 & 1,309 & 7 & 1 & 1,8 \\
\hline Carbono Fixo (\%) & 0,93 & 0,85 & 0,312 & 0,475 & 4 & 1 & 1,8 \\
\hline
\end{tabular}

Rc = coeficiente de correlação da calibração; Rcv = coeficiente de correlação da validação cruzada; SEC = erro padrão da calibração; $\mathrm{SECV}=$ erro padrão da validação cruzada; $\mathrm{RPD}=$ relação de desempenho do desvio. 
Os valores obtidos para os modelos calibrados para os teores de materiais voláteis e carbono fixo apresentaram resultados muito próximos, coerentes com a elevada correlação entre essas duas características do carvão vegetal.

Tais valores mostram a importância dos resultados obtidos nesta pesquisa, indicando que é possível estimar propriedades do carvão a partir dos espectros adquiridos da madeira antes do processo de pirólise.

Andrade (2009), estudando clones de Eucalyptus spp., observou que as calibrações feitas para as propriedades do carvão vegetal a partir dos espectros da madeira sólida, antes de ser carbonizada, não geraram resultados satisfatórios, exceto para a predição do rendimento em carbono fixo, que apresentou valor de correlação igual a 0,74 .

Campos (2008) mostrou que a faixa de 7.140 a $3.500 \mathrm{~cm}^{-1}$ apresentou os melhores desempenhos para validar o modelo de predição do teor de materiais voláteis do carvão. Barcellos (2007), para carvão de Eucalyptus urophylla, encontrou maiores coeficientes de correlação para os teores de materiais voláteis $(0,96)$ e carbono fixo $(0,97)$, no intervalo de 5.260 a $5.000 \mathrm{~cm}^{-1}$.

\section{CONCLUSÕES}

Os resultados indicam que os resíduos de madeira das espécies cardeiro (Scleronema micranthum Ducke, Bombacaceae), cedrinho (Scleronema sp., Bombacaceae), louro (Ocotea sp, Lauraceae) e piquiarana (Caryocar villosum Pers., Caryocaraceae) podem ser utilizados como fonte de energia renovável, uma vez que, por meio do manejo florestal, é possível ter produção de madeira energética sustentada. Entretanto, as melhores espécies para tal finalidade foram o cardeiro e o piquiarana, devido à maior densidade básica, e o louro, com maior teor de lignina e carbono fixo, e, consequentemente, maior poder calorífico.

A técnica NIRS apresentou-se como uma ferramenta que pode ser utilizada para a calibração das propriedades da madeira e do carvão vegetal destas espécies nativas. Podem-se também estimar propriedades do carvão a partir dos espectros adquiridos da madeira antes do processo de pirólise.

\section{AGRADECIMENTOS}

Ao Pesquisador Basílio Frasco Vianez, do Instituto Nacional de Pesquisas da Amazônia (INPA), e ao Professor Setsuo Iwakiri, pela doação dos resíduos de madeira das espécies florestais utilizadas neste trabalho.

\section{STATUS DA SUBMISSÃO}

Recebido: 17 maio., 2013

Aceito: 28 jul., 2014

Publicado: 21 nov., 2014

\section{AUTOR(ES) PARA CORRESPONDÊNCIA}

\section{Vanessa Coelho Almeida}

Departamento de Engenharia e Tecnologia Florestal, Universidade Federal do Paraná UFPR, CEP 80210-170, Curitiba, PR, Brasil e-mail: vanessaufrrj@yahoo.com.br

\section{REFERÊNCIAS}

Andrade CR. Espectroscopia no infravermelho próximo para predizer propriedades da madeira e do carvão de plantio clonal de Eucalyptus sp [dissertação]. Lavras: Universidade Federal de Lavras; 2009.

Associação Brasileira de Normas Técnicas - ABNT. NBR - 8112: Análise imediata: material volátil, cinzas, carbono fixo. São Paulo: ABNT; 1983.

Associação Brasileira de Normas Técnicas - ABNT. NBR 11941-02: determinação da densidade básica em madeira. Rio de Janeiro; 2003.

Associação Brasileira de Normas Técnicas - ABNT. NBR 14660: Madeira - Amostragem e preparação para análise. São Paulo: ABNT; 2004.

Associação Brasileira de Normas Técnicas - ABNT. NBR 7989: Pasta celulósica e madeira - Determinação de lignina insolúvel em ácido. São Paulo: ABNT; 2010a.

Associação Brasileira de Normas Técnicas - ABNT. NBR 14853: Madeira - Determinação do material solúvel em etanol-tolueno e em diclorometano e em acetona. São Paulo: ABNT; 2010b.

Barcellos DC. Caracterização do carvão vegetal através do uso de espectroscopia no infravermelho próximo [dissertação]. Viçosa: Universidade Federal de Viçosa; 2007. 
Brereton RG. Introduction to multivariate calibration in analytical chemistry. Analyst (London) 2000; 125(11): 2125-2154. http://dx.doi.org/10.1039/b003805i.

Campos A. Carvão de Eucalyptus: efeito dos parâmetros da pirólise sobre a madeira e seus componentes químicos e predição da qualidade pela espectroscopia NIR [dissertação]. Lavras: Universidade Federal de Lavras; 2008 .

Foelkel CEB. As biorrefinarias integradas no setor brasileiro de fabricação de celulose e papel de eucalitpo. Eucalyptus Online Book \& Newsletter; 2012. [cited 2012 set.]. Available from: http://www.eucalyptus.com. br/index_eng.html

Kelley SS, Rials TG, Groom LR, So C-L. Use of near infrared spectroscopy to predict the mechanical properties of six softwoods. Holzforschung 2005; 58(3): 252-260.

Martens H, Naes T. Multivariate calibration. New York: Wiley, 1996.

Meder R, Gallagher S, Mackie KL, Böhler H, Meglen RR. Rapid determination of the chemical composition and density of Pinus radiata by PLS modelling of transmission and diffuse reflectance FTIR Spectra. Holzforschung 1999; 53: 261-266.

Oliveira AC, Carneiro ACO, Vital BR, Almeida W, Pereira BLC, Cardonso MT. Parâmetros de qualidade da madeira e do carvão vegetal de Eucalyptus pellita F. Muell. Scientia Forestalis 2010; 38(87): 431-439.

Oliveira JB, Gomes PA, Almeida MR. Caracterização do processo de fabricação de carvão em fornos de alvenaria. In: Penedo WR, editor. Carvão vegetal: destilação, carvoejamento, propriedades, controle de qualidade. Belo Horizonte: CETEC; 1982.

Pastore TCM, Okino EYA, Pastore Júnior FP. Carbonização de madeiras da Amazônia. Parte 1: Floresta Nacional do Tapajós. Brasília: IBAMA-LPF; 1989. Série técnica vol. 12

Pereira BLC, Oliveira AC, Carvalho AMML, Carneiro ACO, Santos LC, Vital BR. Quality of Wood and Charcoal from Eucalyptus Clones for Ironmaster Use. International Journal of Forestry Research 2012; 2012: 1-8. http://dx.doi.org/10.1155/2012/523025.

Petroff G, Doat J. Pyrolyse des bois tropicaux: influence de la composition chimique des bois sur les produits de distillation. Revue Bois et Forêst Tropiques 1978; 177: 51-64.

Protásio TP, Santana JDP, Guimarães Neto RM, Guimarães Júnior JB, Trugilho PF, Ribeiro IB. Avaliação da qualidade do carvão vegetal de Qualea parviflora. Pesquisa Florestal Brasileira 2011; 31(68): 295-307. http://dx.doi.org/10.4336/2011.pfb.31.68.295.
Protásio TP, Trugilho PF, Neves TA, Vieira CMM. Análise de correlação canônica entre características da madeira e do carvão vegetal de Eucalyptus. Scientia Forestalia 2012; 40(95): 317-326.

Savitzky A, Golay MJE. Smoothing and differentiation of data by simplified least-squares procedures. Analytical Chemistry 1964; 36(8): 1627-1639. http:// dx.doi.org/10.1021/ac60214a047.

Schimleck LR, Doran JC, Rimbawanto A. Near infrared spectroscopy for cost effective screening of foliar oil characteristics in a Melaleuca cajuputi breeding population. Journal of Agricultural and Food Chemistry 2003 April; 51(9):2433-2437. http://dx.doi.org/10.1021/ jf020981u. PMid:12696917

Schimleck LR, Evans R. Estimation of Pinus radiata D. Don tracheid morphological characteristics by near infrared spectroscopy. Holzforschung 2004; 58: 66-73.

Schimleck LR, Michell AJ, Raymond CA, Muneri A. Estimation of basic density of Eucalyptus globulus using near-infrared spectroscopy. Canadian Journal of Forest Research 1999; 29(2): 194-201. http://dx.doi. org/10.1139/X98-204.

Silva, AP. Produção de briquetes dos carvões de casca, maravalha e serragem de eucalipto (Eucalyptus pellita), pirolisados às temperaturas máximas de $400^{\circ} \mathrm{C}$ e $600^{\circ} \mathrm{C}$ [monografia]. Seropédica: Universidade Federal Rural do Rio de Janeiro; 2007.

Trugilho PF, Silva DA. Influência da temperatura final de carbonização nas características físicas e químicas do carvão vegetal de jatobá (Himenea courbaril L.). Scientia Agrária 2001; 2(1-2): 45-53.

Trugilho PF, Regazzi AJ, Vital BR, Gomide JL. Aplicação de algumas técnicas multivariadas na avaliação da qualidade da madeira de Eucalyptus e seleção de genótipos superiores para a produção de carvão vegetal. Revista Árvore 1997; 21(1): 113-130.

Vale AT, Abreu VLS, Gonçales JC, Costa AF. Estimativa do poder calorífico superior do carvão vegetal de madeiras de Eucalyptus grandis em função do teor de carbono fixo e do teor de materiais voláteis. Brasil Florestal 2002; 21(73): 47-52.

Vale, AT, Dias, IS, Santana, MAE. Relações entre propriedades químicas, físicas e energéticas da madeira de cinco espécies de cerrado. Ciência Florestal 2010; 36(1): 137-145

Vindaurre GB, Carneiro ACO, Vital BR, Santos RC, Valle MLA. Propriedades energéticas da madeira e do carvão de paricá (Schizolobium amazonicum). Revista Arvore 2012; 36(2): 365-371. http://dx.doi.org/10.1590/ S0100-67622012000200018.

Williams P, Norris K. Near-infrared technology. 2. ed. Saint Paul: American Association of Cereal Chemistry; 2001. 